International Journal of Pure and Applied Mathematics

Volume 98 No. 4 2015, 491-502

ISSN: $1311-8080$ (printed version); ISSN: 1314-3395 (on-line version)

url: http://www.ijpam.eu

doi: http://dx.doi.org/10.12732/ijpam.v98i4.8

ijpam.eu

\title{
ON THE SOLUTIONS OF NON-LINEAR TIME-FRACTIONAL GAS DYNAMIC EQUATIONS: AN ANALYTICAL APPROACH
}

\author{
Olaniyi Samuel Iyiola \\ Department of Mathematics and Statistic \\ King Fahd University of Petroleum and Minerals \\ Dhahran Dammam, SAUDI ARABIA
}

\begin{abstract}
We consider non-linear homogeneous and non-homogeneous gas dynamic equations of time-fractional type in this paper. The approximate solutions of these equations are calculated in the form of series obtained by q-Homotopy Analysis Method (q-HAM). Exact solution is obtained for timefractional homogeneous case while for the case of time-fractional non-homogeneous, exact solution is possible for special case. This is due to the ability to control the auxiliary parameter $h$ and the fraction factor present in this method. The presence of fraction-factor in this method gives it an edge over other existing analytical methods for non-linear differential equations. Comparisons are made with several other analytical methods.
\end{abstract}

AMS Subject Classification: 35Q35, 65M99

Key Words: gas dynamic equation, fractional derivative, non-homogeneous, q-homotopy analysis method

\section{Introduction and Preliminaries}

Calculus of non-integer order is increasingly being used to model physical systems. Caputo [2] for example used the modified form of the Darcys law to 
incorporates the memory term in order to model transport through porous media. Other applications are: control theory of dynamical systems, electrical networks, ground water flow, astrophysics, meteorology, reactive flows and semiconductors see [12] and also [5], [7] for some detailed work on fractional differential equations.

Gas dynamics equations are mathematical expressions that are based on the physical laws of conservation such as the conservation of momentum, laws of conservation of mass, conservation of energy etc. The non-linear equations of ideal gas dynamics are applicable for three types of non-linear waves like refractions, shock fronts and contact discontinuities see [16].

Generally, in such related models, one has to solve a fractional PDE. Analytical methods commonly used to obtain solutions of these kind of non-linear equations have very restricted applications and the numerical techniques give rise to rounding of errors. Therefore, many analytical methods have been put to use successfully to obtain solutions of classical gas dynamics equations such as Adomian Decomposition Method (ADM) [15], Variational Iteration Method (VIM) [13], Homotopy Perturbation Method (HPM) [1], [15] and the timefractional type was considered using differential transform method (DTM) in [3]. Recently, a modified HAM called q-Homotopy Analysis Method was introduced in [4], see also [6], [8], [9], [10]. It was proven that the presence of fraction factor in this method enables a fast convergence compared to the usual HAM. This makes the method more reliable.

In this paper, we apply the q-HAM to initial value problems of the timefractional homogeneous and non-homogeneous gas dynamics equations. Our aim is to exploit the simple, natural and efficient nature of the so called relatively new analytical method (q-HAM) to obtain analytical solutions of the equations considered and exact solution where possible. Finally, we compare the applicability and performance of this method with the exact solutions for some special cases and the solutions obtained by other existing methods in the literature. Numerical results are obtained using Mathematica 9 and MATLAB R2012b.

Caputo's fractional derivative is adopted in this work.

Definition 1.1. The Riemann-Liouville's $(R L)$ fractional integral operator of order $\alpha \geq 0$, of a function $f \in L^{1}(a, b)$ is given as [14]

$$
I^{\alpha} f(t)=\frac{1}{\Gamma(\alpha)} \int_{0}^{t}(t-\tau)^{\alpha-1} f(\tau) d \tau, \quad t>0, \quad \alpha>0
$$

where $\Gamma$ is the Gamma function and $I^{0} f(t)=f(t)$. 
Definition 1.2. The fractional derivative in the Caputo's sense is defined as [14],

$$
\mathcal{D}^{\alpha} f(t)=I^{n-\alpha} D^{n} f(t)=\frac{1}{\Gamma(n-\alpha)} \int_{0}^{t}(t-\tau)^{n-\alpha-1} f^{(n)}(\tau) d \tau,
$$

where $n-1<\alpha \leq n, n \in \mathbb{N}, t>0$.

Lemma 1.1. [14] Let $t \in(a, b]$. Then

$$
\left[I^{\alpha}(t-a)^{\beta}\right](t)=\frac{\Gamma(\beta+1)}{\Gamma(\beta+\alpha+1)}(t-a)^{\beta+\alpha}, \quad \alpha \geqslant 0, \quad \beta>0 .
$$

Definition 1.3. [14] The generalized Mittag-Leffler function in two parameters $\alpha$ and $\beta$ is given as

$$
E_{\alpha, \beta}(z)=\sum_{k=0}^{\infty} \frac{z^{k}}{\Gamma(\alpha k+\beta)}, \quad z, \beta \in \mathbb{C} \quad \text { and } \quad \operatorname{Re}(\alpha)>0 .
$$

\section{2. q-Homotopy Analysis Method (q-HAM)}

Differential equation of the form

$$
N\left[\mathcal{D}_{t}^{\alpha} u(x, t)\right]-f(x, t)=0
$$

is considered, where $N$ is a non-linear operator, $\mathcal{D}_{t}^{\alpha}=\frac{\partial^{\alpha}}{\partial t^{\alpha}}$ denote the Caputo fractional derivative, $(x, t)$ are independent variables, $f$ is a known function and $u$ is an unknown function. To generalize the original homotopy method, the zeroth-order deformation equation is constructed as

$$
(1-n q) L\left(\phi(x, t ; q)-u_{0}(x, t)\right)=q h H(x, t)\left(N\left[\mathcal{D}_{t}^{\alpha} \phi(x, t ; q)\right]-f(x, t)\right)
$$

where $n \geqslant 1, q \in\left[0, \frac{1}{n}\right]$ denotes the so-called embedded parameter, $L$ is an auxiliary linear operator, $h \neq 0$ is an auxiliary parameter, $H(x, t)$ is a non-zero auxiliary function.

It is clearly seen that when $q=0$ and $q=\frac{1}{n}$, equation (6) becomes

$$
\phi(x, t ; 0)=u_{0}(x, t) \quad \text { and } \quad \phi\left(x, t ; \frac{1}{n}\right)=u(x, t)
$$


respectively. So, as $q$ increases from 0 to $\frac{1}{n}$, the solution $\phi(x, t ; q)$ varies from the initial guess $u_{0}(x, t)$ to the solution $u(x, t)$.

If $u_{0}(x, t), L, h, H(x, t)$ are chosen appropriately, solution $\phi(x, t ; q)$ of equation(6) exists for $q \in\left[0, \frac{1}{n}\right]$.

Expansion of $\phi(x, t ; q)$ in Taylor series gives

$$
\phi(x, t ; r)=u_{0}(x, t)+\sum_{m=1}^{\infty} u_{m}(x, t) q^{m} .
$$

where

$$
u_{m}(x, t)=\left.\frac{1}{m !} \frac{\partial^{m} \phi(x, t ; q)}{\partial q^{m}}\right|_{q=0} .
$$

Assume that the auxiliary linear operator $L$, the initial guess $u_{0}$, the auxiliary parameter $h$ and $H(x, t)$ are properly chosen such that the series 8 converges at $q=\frac{1}{n}$, then we have

$$
u(x, t)=u_{0}(x, t)+\sum_{m=1}^{\infty} u_{m}(x, t)\left(\frac{1}{n}\right)^{m} .
$$

Let the vector $u_{n}$ be define as follows:

$$
\vec{u}_{n}=\left\{u_{0}(x, t), u_{1}(x, t), \cdots, u_{n}(x, t)\right\} .
$$

Differentiating equation (6) $m$-times with respect to the (embedding) parameter $q$, then evaluating at $q=0$ and finally dividing them by $m$ !, we have what is known as the $m^{t h}$-order deformation equation ([11]) as

$$
L\left[u_{m}(x, t)-\chi_{m}^{*} u_{m-1}(x, t)\right]=h H(x, t) \mathcal{R}_{m}\left(\vec{u}_{m-1}\right) .
$$

with initial conditions

$$
u_{m}^{(k)}(x, 0)=0, \quad k=0,1,2, \ldots, m-1 .
$$

where

$$
\mathcal{R}_{m}\left(\vec{u}_{m-1}\right)=\left.\frac{1}{(m-1) !} \frac{\partial^{m-1}\left(N\left[\mathcal{D}_{t}^{\alpha} \phi(x, t ; q)\right]-f(x, t)\right)}{\partial q^{m-1}}\right|_{q=0}
$$

and

$$
\chi_{m}^{*}= \begin{cases}0 & m \leqslant 1 \\ n & \text { otherwise }\end{cases}
$$




\section{The Time-Fractional Homogeneous Gas Dynamic Equation}

We consider the time fractional homogeneous gas dynamic equation. Let

$$
\frac{\partial^{\alpha} u}{\partial t^{\alpha}}+\frac{1}{2} \frac{\partial\left(u^{2}\right)}{\partial x}-u(1-u)=0, \quad 0 \leqslant x \leqslant 1, \quad t>0, \quad 0<\alpha \leq 1
$$

subjected to the initial condition

$$
u(x, 0)=a e^{-x}
$$

The exact solution to this problem, when $a=1$ and $\alpha=1$, is

$$
u(x, t)=e^{t-x}
$$

Many authors have worked on this problem when $\alpha=1$, using various methods see [1], [3], [13], [15].

\subsection{Application of q-HAM}

In order to use q-HAM to solve the problem considered in (16), we choose the linear operator

$$
L[\phi(x, t ; q)]=\mathcal{D}_{t}^{\alpha} \phi(x, t ; q)
$$

with property that $L\left[c_{1}\right]=0, c_{1}$ is constant.

We use initial approximation $u_{0}(x, t)=a e^{-x}$. We can then define the nonlinear operator as

$$
N[\phi(x, t ; q)]=\mathcal{D}_{t}^{\alpha} \phi(x, t ; q)+\phi(x, t ; q) \phi_{x}(x, t ; q)-\phi(x, t ; q)+(\phi(x, t ; q))^{2} .
$$

We construct the zeroth order deformation equation

$$
(1-n q) L\left[\phi(x, t ; q)-u_{0}(x, t)\right]=q h H(x, t) N\left[\mathcal{D}_{t}^{\alpha} \phi(x, t ; q)\right]
$$

We choose $H(x, t)=1$ to obtain the mth-order deformation equation to be

$$
L\left[u_{m}(x, t)-\chi_{m}^{*} u_{m-1}(x, t)\right]=h \mathcal{R}_{m}\left(\vec{u}_{m-1}\right),
$$

with initial condition for $m \geqslant 1, u_{m}(x, 0)=0, \chi_{m}^{*}$ is as defined in (15) and

$$
\mathcal{R}_{m}\left(\vec{u}_{m-1}\right)=\mathcal{D}_{t}^{\alpha} u_{m-1}+\sum_{k=0}^{m-1} u_{k}\left(u_{m-1-k}\right)_{x}-u_{m-1}+\sum_{k=0}^{m-1} u_{k} u_{m-1-k}
$$


So, the solution to the equation (16) for $m \geqslant 1$ becomes

$$
u_{m}(x, t)=\chi_{m}^{*} u_{m-1}+h I_{t}^{\alpha}\left[\mathcal{R}_{m}\left(\vec{u}_{m-1}\right)\right] .
$$

We therefore obtain components of the solution using q-HAM successively as follows

$$
\begin{aligned}
u_{1}(x, t)= & \chi_{1}^{*} u_{0}+h I_{t}^{\alpha}\left[\mathcal{D}_{t}^{\alpha} u_{0}+u_{0}\left(u_{0}\right)_{x}-u_{0}+u_{0}^{2}\right] \\
= & -a h e^{-x} \frac{t^{\alpha}}{\Gamma(1+\alpha)} \\
u_{2}(x, t)= & \chi_{2}^{*} u_{1}+h I_{t}^{\alpha}\left[\mathcal{D}_{t}^{\alpha} u_{1}+u_{0}\left(u_{1}\right)_{x}+u_{1}\left(u_{0}\right)_{x}-u_{1}+2 u_{0} u_{1}\right] \\
= & (n+h) u_{1}+a h^{2} e^{-x} \frac{t^{2 \alpha}}{\Gamma(1+2 \alpha)} \\
u_{3}(x, t)= & \chi_{3}^{*} u_{2}+h I_{t}^{\alpha}\left[\mathcal{D}_{t}^{\alpha} u_{2}+u_{0}\left(u_{2}\right)_{x}+u_{1}\left(u_{1}\right)_{x}\right. \\
& \left.+u_{2}\left(u_{0}\right)_{x}-u_{2}+2 u_{0} u_{2}+u_{1}^{2}\right] \\
= & (n+h) u_{2}+a(n+h) h^{2} e^{-x} \frac{t^{2 \alpha}}{\Gamma(1+2 \alpha)} \\
& -a h^{3} e^{-x} \frac{t^{3 \alpha}}{\Gamma(1+3 \alpha)} .
\end{aligned}
$$

In the same way, $u_{m}(x, t)$ for $m=4,5, \cdots$ can be obtained Mathematica.

Then the series solution expression by q-HAM can be written in the form

$$
\begin{aligned}
u(x, t ; n ; h)= & a e^{-x}+\sum_{i=1}^{\infty} u_{i}(x, t ; n ; h)\left(\frac{1}{n}\right)^{i} \\
= & a e^{-x}-a h e^{-x} \frac{t^{\alpha}}{n \Gamma(1+\alpha)}+\frac{(n+h) u_{1}}{n}+\frac{(n+h) u_{2}}{n^{2}} \\
& +a h^{2} e^{-x} \frac{t^{2 \alpha}}{n^{2} \Gamma(1+2 \alpha)}+a(n+h) h^{2} e^{-x} \frac{t^{2 \alpha}}{n^{3} \Gamma(1+2 \alpha)} \\
& -a h^{3} e^{-x} \frac{t^{3 \alpha}}{n^{3} \Gamma(1+3 \alpha)}+\cdots .
\end{aligned}
$$

Equation (27) is an appropriate solution to the problem (16) in terms of convergence parameter $h$ and $n$.

Remark 3.1. When $n=1$, we choose appropriate $h=-1$ to obtain

$$
u(x, t)=a e^{-x}+a e^{-x} \frac{t^{\alpha}}{\Gamma(1+\alpha)}+a e^{-x} \frac{t^{2 \alpha}}{\Gamma(1+2 \alpha)}+a e^{-x} \frac{t^{3 \alpha}}{\Gamma(1+3 \alpha)}+\cdots
$$




$$
\begin{aligned}
& =a e^{-x}\left[1+\frac{t^{\alpha}}{\Gamma(1+\alpha)}+\frac{t^{2 \alpha}}{\Gamma(1+2 \alpha)}+\frac{t^{3 \alpha}}{\Gamma(1+3 \alpha)}+\cdots\right] \\
& =a e^{-x} E_{\alpha}\left(t^{\alpha}\right) .
\end{aligned}
$$

Hence, we obtain exact solution of the time-fractional homogeneous gas dynamic equation (16). For $\alpha=1$, we equally get the exact solution of the classical homogeneous gas dynamic equation which

$$
u(x, t)=a e^{t-x} .
$$

Remark 3.2. Observe that infinite sum is required in (27) to obtain the exact solution with $h=-1$ but Figure 1 displays a good approximation by taking $h=-1.2$ with just three terms of the series solution obtained in (27). Figures 2 and 3 give the effect of both $h$ and $n$ on the solution given by q-HAM with just few terms as well.

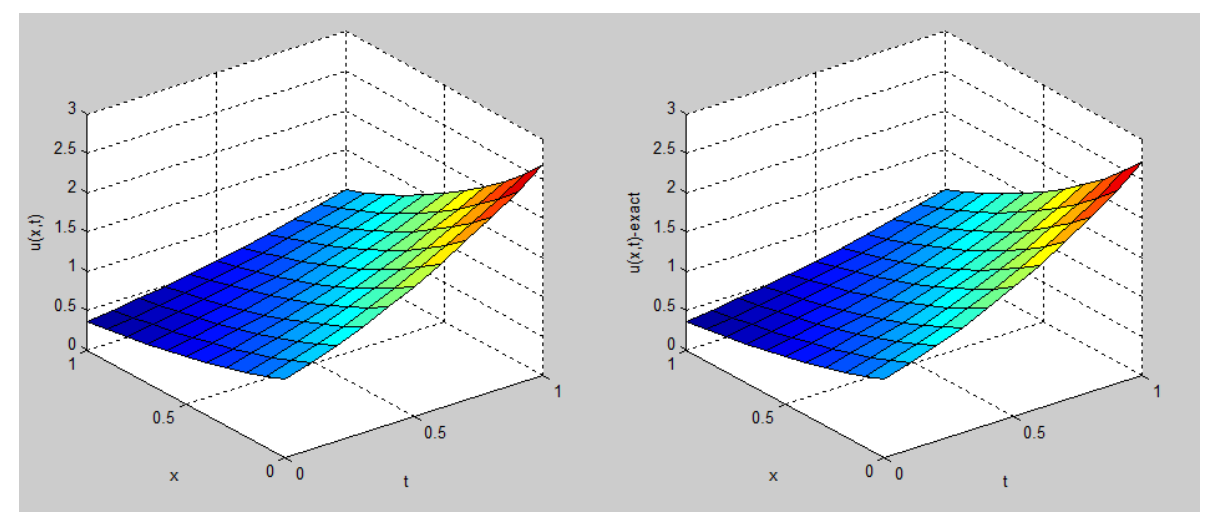

Figure 1: q-HAM solution plot of $u$ for $h=-1.2, n=1, a=1$ and $\alpha=1$ against exact solution

\section{The Time-Fractional Non-Homogeneous Gas Dynamic Equation}

We consider the time fractional non-homogeneous gas dynamics equation. Let

$$
\frac{\partial^{\beta} u}{\partial t^{\beta}}+\frac{1}{2} \frac{\partial\left(u^{2}\right)}{\partial x}-u(1-u)=-e^{t-x}, \quad 0 \leqslant x \leqslant 1, \quad t>0, \quad 0<\beta \leq 1
$$

subjected to the initial condition

$$
u(x, 0)=1-e^{-x} .
$$




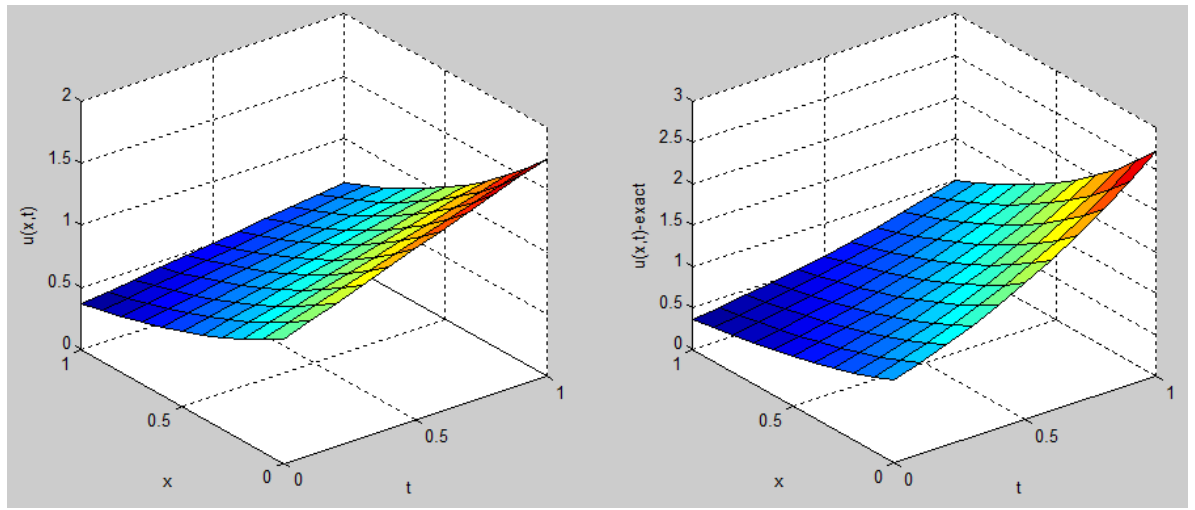

Figure 2: q-HAM solution plot of $u$ for $h=-0.5, n=1, a=1$ and $\alpha=1$ against exact solution

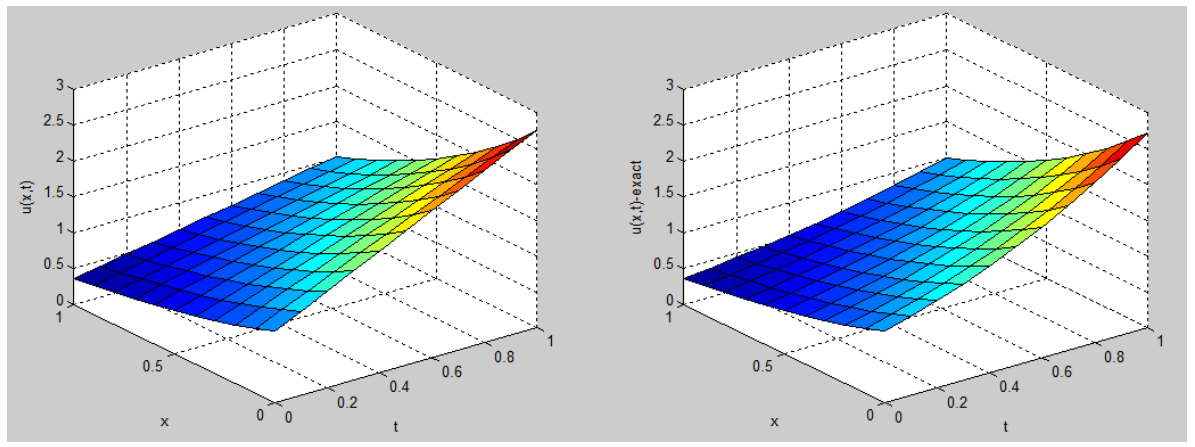

Figure 3: q-HAM solution plot of $u$ for $h=-0.5, n=3, a=1$ and $\alpha=1$ against exact solution

The exact solution to this problem, when $\beta=1$, is

$$
u(x, t)=1-e^{t-x} .
$$

Many authors have worked on this problem when $\beta=1$, using various methods see [1], [3], [13], [15].

\subsection{Application of q-HAM}

We follow the same procedure as in first case using the initial approximation to be $u_{0}(x, t)=1-e^{-x}$. 
We construct the zeroth order deformation equation

$$
(1-n q) L\left[\phi(x, t ; q)-u_{0}(x, t)\right]=q h H(x, t)\left(N\left[\mathcal{D}_{t}^{\beta} \phi(x, t ; q)\right]+e^{t-x}\right) .
$$

We choose $H(x, t)=1$ to obtain the mth-order deformation equation to be

$$
L\left[u_{m}(x, t)-\chi_{m}^{*} u_{m-1}(x, t)\right]=h \mathcal{R}_{m}\left(\vec{u}_{m-1}\right),
$$

with initial condition for $m \geqslant 1, u_{m}(x, 0)=0, \chi_{m}^{*}$ is as defined in (15) and

$$
\mathcal{R}_{m}\left(\vec{u}_{m-1}\right)=\mathcal{D}_{t}^{\beta} u_{m-1}+\sum_{k=0}^{m-1} u_{k}\left(u_{m-1-k}\right)_{x}-u_{m-1}+\sum_{k=0}^{m-1} u_{k} u_{m-1-k}+e^{t-x} .
$$

So, the solution to the equation (30) for $m \geqslant 1$ becomes

$$
u_{m}(x, t)=\chi_{m}^{*} u_{m-1}+h I_{t}^{\beta}\left[\mathcal{R}_{m}\left(\vec{u}_{m-1}\right)\right] .
$$

We therefore obtain components of the solution using q-HAM successively as follows

$$
\begin{aligned}
u_{1}(x, t)= & \chi_{1}^{*} u_{0}+h I_{t}^{\beta}\left[\mathcal{D}_{t}^{\beta} u_{0}+u_{0}\left(u_{0}\right)_{x}-u_{0}+u_{0}^{2}+e^{t-x}\right] \\
= & h e^{-x} t^{\beta} E_{1, \beta+1}(t) \\
u_{2}(x, t)= & \chi_{2}^{*} u_{1}+h I_{t}^{\beta}\left[\mathcal{D}_{t}^{\beta} u_{1}+u_{0}\left(u_{1}\right)_{x}+u_{1}\left(u_{0}\right)_{x}-u_{1}+2 u_{0} u_{1}+e^{t-x}\right] \\
= & (n+h) u_{1}+h e^{-x} t^{\beta} E_{1, \beta+1}(t) \\
u_{3}(x, t)= & \chi_{3}^{*} u_{2}+h I_{t}^{\beta}\left[\mathcal{D}_{t}^{\beta} u_{2}+u_{0}\left(u_{2}\right)_{x}+u_{1}\left(u_{1}\right)_{x}+u_{2}\left(u_{0}\right)_{x}\right. \\
& \left.-u_{2}+2 u_{0} u_{2}+u_{1}^{2}+e^{t-x}\right] \\
= & (n+h) u_{2}+h e^{-x} t^{\beta} E_{1, \beta+1}(t) .
\end{aligned}
$$

In the same way, $u_{m}(x, t)$ for $m=4,5, \cdots$ can be obtained using Mathematica 9.

Then the series solution expression by q-HAM can be written in the form

$$
\begin{aligned}
u(x, t ; n ; h)= & 1-e^{-x}+\sum_{i=1}^{\infty} u_{i}(x, t ; n ; h)\left(\frac{1}{n}\right)^{i} \\
= & 1-e^{-x}+\frac{h e^{-x} t^{\beta} E_{1, \beta+1}(t)}{n}+\frac{(n+h) u_{1}}{n^{2}}+\frac{h e^{-x} t^{\beta} E_{1, \beta+1}(t)}{n^{2}} \\
& \frac{(n+h) u_{2}}{n^{3}}+\frac{h e^{-x} t^{\beta} E_{1, \beta+1}(t)}{n^{3}}+\cdots .
\end{aligned}
$$

Equation (40) is an appropriate solution to the problem (30) in terms of convergence parameter $h$ and $n$. 
Remark 4.1. Using the first two terms of the q-HAM series in (40), when $n=1$ and $\alpha=1$, we choose appropriate $h=-1$, we obtain

$$
\begin{aligned}
u(x, t) & =1-e^{-x}+t e^{-x} E_{1,2}(t) \\
& =1-e^{-x}-e^{t-x}+e^{-x} \\
& =1-e^{t-x} .
\end{aligned}
$$

Hence, we obtain exact solution to the non-homogeneous gas dynamic equation (30) given by just two terms of the series.

Remark 4.2. Similar numerical comparisons can also be made with the exact solution as in the homogeneous case.

\section{Conclusion}

The major achievement of this paper is the demonstration of the successful application of the q-HAM to obtain analytical solutions of the non-linear gas dynamic equations of time-fractional type. Exact solution is obtained in the case of time-fractional homogeneous gas dynamic equation just by choosing an appropriate auxiliary parameter $h$. This choice of $h$ is generally determined by what is called $h$-curve. Our results confirm that the method of solution used is really effective for handling solutions of a class of non-linear Partial Differential Equations of fractional order system. Considering the results obtained by other analytical methods such as HPM, VIM, ADM, DTM etc., the accuracy of Homotopy Analytical Method is clearly seeing in the sense that just two terms are needed in the case of the non-homogeneous gas dynamic equation unlike other methods.

\section{Acknowledgement}

The financial support received from King Fahd University of Petroleum and Minerals (KFUPM) is gratefully acknowledged.

\section{References}

[1] H. Aminikhah and A. Jamalian; Numerical Approximation for Nonlinear Gas Dynamic Equation, Int. J. of Partial Diff. Equations, vol. (2013), Article ID 846749, 7 pages. http://dx.doi.org/10.1155/2013/846749 
[2] M. Caputo; Diffusion of fluids in porous media with memory, Geothermics, 28, (1999), 113-130. http://dx.doi.org/10.1016/S0375-6505(98)00047-9

[3] S. Das and R. Kumar; Approximate analytical solutions of fractional gas dynamic equations, Applied Mathematics and Computation, 217, (2011) 9905-9915. doi:10.1016/j.amc.2011.03.144

[4] M.A. El-Tawil and S. N. Huseen; The Q-Homotopy Analysis Method (QHAM), Int. J. of Appl. Math. and Mech., 8, No. 15, (2012), 51-75. Y2012V8N15P51C52708072.PDF

[5] K. A. Gepreel and A. A. Al-Thobaiti; Exact solutions of nonlinear partial fractional differential equations using fractional sub-equation method, Indian J Phys, 88, No. 3, (2014), 293300. doi:10.1007/s12648-013-0407-0

[6] O. S. Iyiola; A Numerical Study of Ito Equation and Sawada-Kotera Equation both of Time-fractional Type, Adv. Math: Sci Journal, 2, No. 2, (2013), 71-79. http://repository.research-publication.com/id/eprint/36

[7] O. S. Iyiola; Solving $k$-Fractional Hilfer Differencial Equations via Combined Fractional Integral Transform Methods, British J. of Maths and Computer Science, 4, No. 10, (2014), 1427-1436. DOI : 10.9734/BJMCS/2014/9444

[8] O. S. Iyiola and G. O. Ojo; Analytical Solutions of Time-fractional Models for Homogeneous Gardner Equation and Non-homogeneous Differential Equations, Ain Shams Engineering Journal, 5, (2014), 999-1004. doi:10.1016/j.asej.2014.03.014

[9] O. S. Iyiola and G. O. Ojo; On the Analytical Solution of FornbergWhitham Equation with the new Fractional Derivative, Pramana-Journal of Physics (2014), In press.

[10] O. S. Iyiola and F.D. Zaman; A Fractional Diffusion Equation Model for Cancer Tumor, AIP Advances, 4, (2014), 107121. http://dx.doi.org/10.1063/1.4898331

[11] S.-J. Liao; An approximate solution technique not depending on small parameters: a special example, International Journal of Non-linear Mechanics, 30, No. 3, (1995), 371-380. doi:10.1016/0020-7462(94)00054-E

[12] G.I. Marchuk; Mathematical Models in Environmental Problems, NorthHolland, Elsevier Science Publisher, (1986). 
[13] M. Matinfar, M. Saeidy, M. Mahdavi, and M. Razaei; Variational iteration method for exact solution of gas dynamic equation using He's polynomials, Bulletin of Mathematical Analysis and Applications, 3, No. 3, (2011), 5055. http://91.187.98.171/bmathaa/vol3issue3.html

[14] I. Podlubny; Fractional Differential Equations, Vol. 198 of Mathematics in Science and Engineering, Academic Press, San Diego, Calif, USA, (1999).

[15] J. Singh, D. Kumar and A Kilicman; Homotopy perturbation method for fractional gas dynamics eqaution using Sumudu transform, Abstract and Applied Analysis, vol. (2013), Article ID 934060. http://dx.doi.org/10.1155/2013/934060

[16] J. L. Steger and R.F. Warming; Flux vector splitting of the inviscid gas dynamic equations with application to finite-difference methods, J. Comput. Phys., 40, (1981) 263293. doi:10.1016/0021-9991(81)90210-2 